Sharif University of Technology
Scientia Iranica
SCIENTIA
I RAN I CA
http://scientiairanica.sharif.edu

\title{
A numerical investigation of the effect of the temperature on the seepage calculation
}

\author{
A. Tabarsa* and M. Lashkarbolok \\ Faculty of Engineering, Golestan University, Gorgan, Iran.
}

Received 8 October 2015; received in revised form 21 September 2016; accepted 4 February 2017

\author{
KEYWORDS \\ Temperature \\ distribution; \\ Meshfree method; \\ Seepage; \\ Permeability; \\ Least squares.
}

\begin{abstract}
Temperature difference in the soil and its environments is a common phenomenon. Soil permeability changes in parallel to temperature, mostly due to water viscosity variations in different temperatures. A more realistic estimation of the seepage value through and beneath hydraulic structures leads to their more efficient design. In this paper, the heat conduction equation is solved by the least-squares mesh-free method to calculate the temperature distribution in soil. Distribution of permeability coefficients can vary irregularly and may lead to some difficulties in mesh-based methods. In these methods, soil permeability changes in each mesh, and finer mesh or some kind of interpolation is required to contribute to the solution procedure. Since there is no need to form elements or grids in a mesh-free method, it can handle this irregular variation simply. Herein, the seepage equation is solved by the same least squares mesh-free method. The method is integral-free, simple, and efficient in calculation due to its sparse and positive definite matrices. The scheme is validated by solving a simplified version of the governing equations. Problems that are more complicated are dealt with to investigate the phenomenon numerically.
\end{abstract}

(C) 2018 Sharif University of Technology. All rights reserved.

\section{Introduction}

Soil thermal properties are of great importance in many engineering projects and other situations where heat transfer takes place in the soil. The problem of heat transfer in soils is very complicated since thermal properties of a soil layer depend on many parameters, which may not be constant through time cycles [1]. Heat transfer processes were studied through modelling of soil temperature. The results revealing an increase in frequency or magnitude of infiltration events could mimic simple spring time surface warming [2]. In general, heating is an effective method of soil treatment

*. Corresponding authors. Tel.: +98 1732254160 E-mail addresses: a.tabarsa@gu.ac.ir (A. Tabarsa); m.lbolok@gu.ac.ir (M. Lashkarbolok)

doi: $10.24200 /$ sci.2018.20421 for only fine-grained (clayey) soils. High temperatures induce permanent physical reactions in the clay minerals, as well as a drying effect by water evaporation [3]. High temperatures affect particle size distribution, mass loss, mineralogy, and permeability of the soil. In sandy soils, the particle size decreases as temperature increases due to the mobilization of fines, which is likely to occur due to the bonding of fines to sand grains affected by temperature [4]. In clayey soils, the overall particle size increases as temperature increases due to aggregation and cementation of the clay fraction [4]. Since the thermal expansion coefficient of the soil grains is low (in the order of $10^{-5} \frac{1}{{ }^{\circ} \mathrm{C}}$ ), changes in the particle sizes are not significant in the permeability of soils in natural environmental temperatures. However, particle size may be considered in the thermal modification of soils. The value of the seepage discharge through the foundation of hydraulic structures is of great importance in a design procedure. This discharge depends 
on the hydraulic gradients, permeability coefficients, and geometry of the structure. Distribution of the temperature in the soil layer affects the value of the permeability coefficients. Cho et al. reported that the influence of temperature on the permeability of bentonite is mainly due to the viscosity and density of water [5]. Towhata et al. showed that any change in water viscosity with temperature is the most important mechanism to modify soils' properties [6]. The same results were obtained by the study of Villar and Lioret [7]. Romero et al. investigated the effect of temperature on the soil permeability in unsaturated clay [8]. Ye et al. demonstrated that hydraulic conductivity increases as temperature increases [9]. Stetyukha simulated changes in the thermal condition of soils under the effect of channel change of a riverbed numerically [10]. The thermal influence of the warm oil pipeline on the permafrost foundation was simulated using a developed coupled temperature seepage mathematical model and the finite-element method [11]. Yousefi et al. studied leakage in embankment dam by applying seepage and thermal numerical simulations using a finiteelement method [12]. Cui et al. presented the coupled thermo-hydraulic boundary governing formulation as well as the coupled thermo-hydraulic boundary condition, which could be implemented in a finite element method [13]. Numerical analyses using the Finite Difference Method (FDM) were limited to cases where the calculation domains are comparatively simple [14]. Fukuchi applied Interpolation FDM (IFDM) to solve two-dimensional elliptic partial differential equations over complex domains for the steady-state seepage problem [14].

In the present study, a mesh-free method is used in the numerical calculation of the seepage problem considering thermal variations in a soil layer. Since permeability coefficients at any point in the soil will change in accordance with the temperature, the distribution of the permeability coefficient may vary at any point in the soil. In this situation, it is simpler to use a method that discretizes the domain of the problem by some scatter nodes, instead of elements. Mesh-free methods are rather new approaches to solving partial differential equations. The main feature of these methods is that they do not require mesh or grid in domain discretization. These methods need node generation instead of mesh generation. A detailed review of the mesh-free methods was provided by Belytschko [15], Liu [16], and Liu and Gu [17]. Ding et al. [18] presented the earliest meshless method based on the least squares technique. Collocated Discrete Least Squares (CDLS) is a meshless method based on the least squares technique enjoying symmetric and positive definite properties that make it efficient in calculation and simple in formulation. The main idea of the method is adopted from the least squares technique in a finite-element method. Afshar and Lashckarbolok presented CDLS meshless method along with a posteriori error estimate and an adaptive refinement strategy in conjunction with the method [19]. This method has been used to simulate the free surface [20], Newtonian [21] and nonNewtonian $[22,23]$ flow problems. In this study, the CDLS mesh-free method is applied to the numerical calculation of seepage problem while temperature variation is taken into account. The solution procedure of this method comprises some simple matrix algebra. Since local domains are used, all matrices become sparse and easy to operate. The values of permeability coefficients are stored in a vector and will be considered in the solution procedure without any complexity. The CDLS method is integral-free and produces symmetric and positive definite coefficients matrices that make it suitable for iterative solvers. In this research, first, the heat conduction equation is solved to obtain the temperature distribution in the soil layer. Then, by using the value of the temperature at any point, the permeability coefficients are evaluated and applied to the seepage equation to find the hydraulic heads and velocities. Although all formulations are in a twodimensional space in this research, dealing with threedimensional problems will be straightforward using the proposed pattern. Two problems with different boundary conditions are dealt with to show the ability of the proposed scheme. Herein, a fully vectorized form of the CDLS method is used to solve the problems. In this kind of formulation, the permeability coefficients can be stored in a vector, and some simple matrix algebra is required for the solution procedure.

\section{Governing equations}

The values of the temperatures $(T)$ at each point in the soil $(x, z)$ and at any time $(t)$ must be calculated by solving the heat conduction equation. The equation in Cartesian coordinate $(x-z)$ is given by:

$$
\frac{\partial T}{\partial t}=\alpha \cdot\left(\frac{\partial^{2} T}{\partial x^{2}}+\frac{\partial^{2} T}{\partial z^{2}}\right)
$$

where $\alpha$ is the thermal diffusivity. Typical values of thermal diffusivity for different kinds of soils are given in Table 1.

Table 1. Typical values of thermal diffusivity [3].

\begin{tabular}{cc}
\hline Soil & $\begin{array}{r}\text { Thermal diffusivity } \\
\left(\mathbf{m}^{\mathbf{2}} \mathbf{d a y}^{\mathbf{- 1}}\right)\end{array}$ \\
\hline Sand (or gravel) & 0.039 \\
Silt & 0.05 \\
Clay & 0.046 \\
Loam & 0.042 \\
Saturated sand & 0.079 \\
Saturated silt or clay & 0.056 \\
\hline
\end{tabular}


Table 2. Viscosity of water in different temperatures.

\begin{tabular}{cc}
\hline Temperature $\left({ }^{\circ} \mathbf{C}\right)$ & Viscosity (mPa.s) \\
\hline 10 & 1.308 \\
20 & 1.002 \\
30 & 0.7978 \\
40 & 0.6531 \\
50 & 0.5471 \\
60 & 0.4658 \\
70 & 0.4044 \\
80 & 0.355 \\
90 & 0.315 \\
100 & 0.2822 \\
\hline
\end{tabular}

Based on Darcy law in the seepage velocity in a two-dimensional soil domain and considering that the velocity field is divergence-free, the governing equation of the seepage problem can be stated by the following equation:

$$
\frac{\partial}{\partial x}\left(K_{x} \cdot \frac{\partial h}{\partial x}\right)+\frac{\partial}{\partial z}\left(K_{z} \cdot \frac{\partial h}{\partial z}\right)=0 .
$$

Using chain rule, Eq. (1) can be written by:

$$
\frac{\partial K_{x}}{\partial x} \cdot \frac{\partial h}{\partial x}+K_{x} \cdot \frac{\partial^{2} h}{\partial x^{2}}+\frac{\partial K_{z}}{\partial z} \cdot \frac{\partial h}{\partial z}+K_{z} \cdot \frac{\partial^{2} h}{\partial z^{2}}=0,
$$

where $h, K_{x}$, and $K_{z}$ are the water head, coefficients of permeability in $x$, and the coefficients of permeability in $z$ direction, respectively. To relate the values of the permeability to the temperature, the viscosity of water $(\eta)$ is considered to change in parallel to the temperature. As temperature increases, viscosity decreases, and then the permeability increases. The coefficient of permeability ( $K$ in general) is standardized at $20^{\circ} \mathrm{C}$, and the permeability at any temperature $T$ is related to the permeability at $20^{\circ} \mathrm{C}, K_{20}$, by the following equation:

$$
K_{T}=K_{20} \frac{\eta_{20}}{\eta_{T}}
$$

Herein, the value of the temperature at any position in the soil (which is a time-dependent variable) is calculated by solving Eq. (1). Using these values, the viscosity of water, at any point, can be obtained by the existing experimental data. In this paper, Table 2 is used to interpolate the viscosity of water at different temperatures. The viscosity comes from the cohesive forces among water molecules. This cohesive force is reduced by increasing the temperature, hence reducing the viscosity.

\section{Method formulation}

The formulation of the CDLS method is illustrated comprehensively in the references [19-22]. To solve a problem, the domain and its boundaries must be discretized by nodal (with the number of $n$ ) and

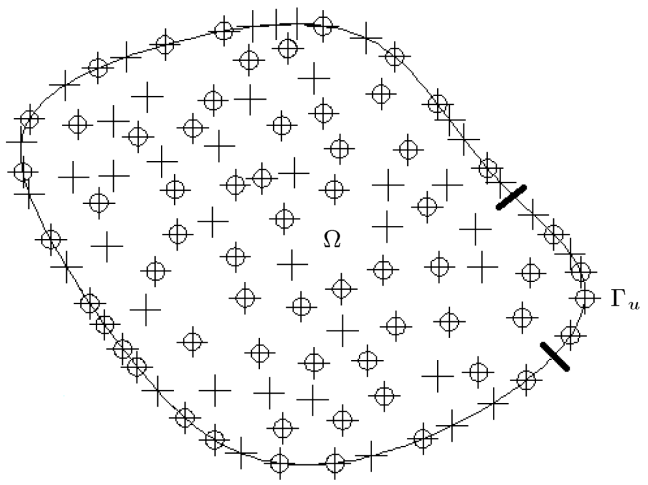

Figure 1. The domain discretized by field nodes (circles) and collocation points (plus sign) [24].

collocation points (with the number of $m$ ) [19]. This kind of discretization is simpler than using mesh, cell or grids, especially when the domain of the problem consists of inhomogeneous materials [19]. To write up the method formulation of solving the governing equations in a matrix format, it is required to form shape functions matrices [22]. The typical form of a shape function matrix is given by:

$$
\varphi=\left[\begin{array}{cccc}
\varphi_{11} & \varphi_{11} & \ldots & \varphi_{1 m} \\
\varphi_{21} & \varphi_{22} & \ldots & \varphi_{2 m} \\
\vdots & \vdots & \vdots & \vdots \\
\varphi_{n 1} & \varphi_{n 2} & \ldots & \varphi_{n m}
\end{array}\right]
$$

where $\varphi_{i j}$ means the value of the $i$ th shape function at the $j$ th collocation point.

As shown in Figure 1, the problem domain and its boundaries are discretized using some scattered field nodes and collocation points [19]. Besides the field nodes, the collocation points are used in the domain of the problem and on its boundaries. In this methodology, in each field node, one collocation point has to be placed, as shown in Figure 1 [22,24]. The approximated value of unknown function, $u$, at a collocation point, $k$, with coordinate $x_{k}$ can be obtained through the following interpolation [19]:

$$
u\left(x_{k}\right)=u_{k}=\sum_{i=1}^{\bar{n}} \varphi_{i_{k}} \cdot u_{i},
$$

where $u_{i}$ is the value of the unknown function at the $i$ th field node. $\bar{n}$ is the number of field nodes that the $k$ th collocation point with coordinate $x_{k}$ can have in its domain $[22,24]$. This idea of compact support is shown in Figure 2. To set up such a domain for each collocation point, radius $d s$ is defined so that a specific number of field nodes can be placed into its support domain $(\bar{n})$ [22]. In Eq. (1), $\varphi_{i k}$ is the value of the shape function of the $i$ th node at the $k$ th collocation point.

In this study, RPIM is used to calculate the value of the shape functions. The detailed descriptions of 


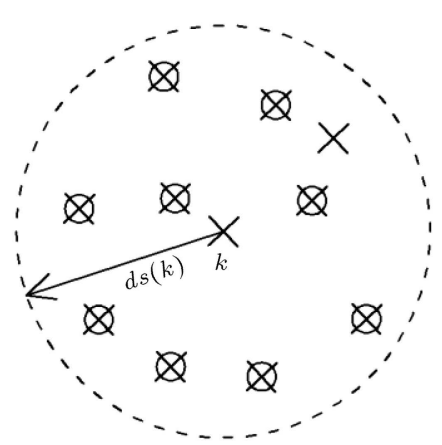

Figure 2. Compact support of the $k$ th collocation [22,24].

the procedure are available elsewhere $[16,17]$. In the following numerical examples, a multi-quadratic radial basis function augmented with completed second-order polynomials is used in the radial point interpolation. Multi-quadratic radial basis function $(q)$ is given by $[16]$ :

$$
q(r)=\left(d s^{2}+r^{2}\right)^{1.03}
$$

where $d s$ is the radius of the compact support domain that is defined by the radius associated with each collocation point so that 20 nodes are placed into the support domain of that collocation.

Since there are first- and second-order operators in the governing equations, it is required to find the first and second derivatives of the shape function matrix, too. They are represented by $\frac{\partial \varphi}{\partial x}, \frac{\partial \varphi}{\partial x}, \frac{\partial^{2} \varphi}{\partial x^{2}}$, and $\frac{\partial^{2} \varphi}{\partial z^{2}}$ here.

In the presented scheme, first, the heat conduction problem is solved over time. An implicit Euler method is applied to the time discretization since it is not required to fulfill any time step size criterion for the stability condition. The stability of the implicit Euler method is proven in many references, such as [25]. It is given by:

$$
T^{n+1}=T^{n}+\alpha \cdot \Delta t \cdot\left(\frac{\partial^{2} T^{n+1}}{\partial x^{2}}+\frac{\partial^{2} T^{n+1}}{\partial z^{2}}\right),
$$

where $T^{n}$ is the value of temperature in the $n$th time step. The matrix formulation of the proposed method in calculating the temperature value at any node is given by:

$$
\begin{aligned}
& L b=\varphi-\alpha \Delta t \cdot\left(\frac{\partial^{2} \varphi}{\partial x^{2}}+\frac{\partial^{2} \varphi}{\partial z^{2}}\right), \\
& K=L b \times L b^{T},
\end{aligned}
$$

where $K$ is the coefficient matrix, which is symmetric and sparse. In this methodology, boundary conditions are satisfied by penalty method as described in [22]. Now, the vector of the temperature values at nodes at $(n+1)$ th time step $\left(T^{n+1}\right)$ is written by:

$$
T^{n+1}=K^{-1} \times F,
$$

where $F$ is the right-hand side vector, obtained after enforcing the boundary conditions. After calculating the temperatures at the desired time, the values of the permeability coefficients at any point can be calculated according to their corresponding temperature using the given data in Table 1 . Then, the hydraulic head can be obtained by solving Eq. (2) using the following formulation:

$$
\begin{aligned}
L b= & \frac{\partial K_{x}}{\partial x} \otimes \frac{\partial \varphi}{\partial x}+K_{x} \otimes \frac{\partial^{2} \varphi}{\partial x^{2}} \\
& +\frac{\partial K_{z}}{\partial z} \otimes \frac{\partial \varphi}{\partial z}+K_{z} \otimes \frac{\partial^{2} \varphi}{\partial z^{2}}, \\
K= & L b \times L b^{T}, \\
h= & K^{-1} \times F .
\end{aligned}
$$

It should be noted that all parameters are sparse matrices, making the calculation efficient. Operator $\otimes$ is defined in the reference [22].

\section{Sample solved problems}

To validate the proposed scheme, a problem with available analytical solution is considered within a simple domain in Problem 1. In Problem 2, the variation of the ambient temperature on the seepage value is investigated at different cases for a typical concrete dam.

\subsection{Problem 1}

Consider a rectangular domain with given boundary conditions in Figure 3. The governing equation in this domain is defined by:

$$
\frac{\partial}{\partial x}\left(\lambda(x, z) \cdot \frac{\partial \Omega}{\partial x}\right)+\frac{\partial}{\partial z}\left(\lambda(x, z) \cdot \frac{\partial \Omega}{\partial z}\right)=0,
$$

where, $\lambda(x, z)$ is defined by:

$$
\lambda(x, z)=x^{2}+2 x z+z^{2}-x-z+0.25 .
$$

These equations mimic the equation that was defined in Eq. (2). Therefore, it can be considered as a suitable

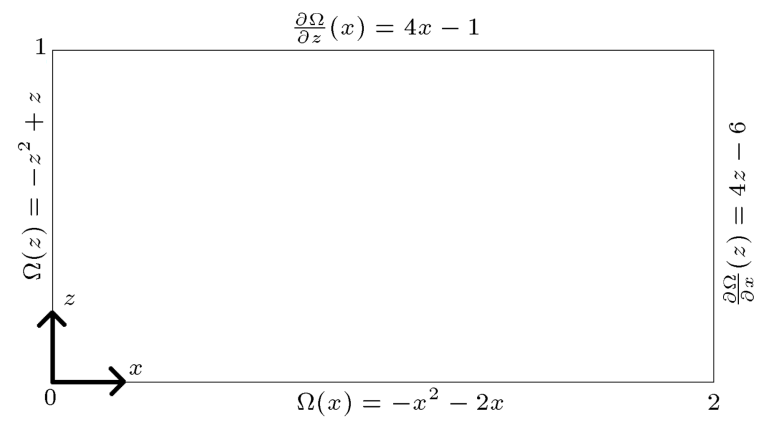

Figure 3. The domain and boundary conditions for Problem 1. 


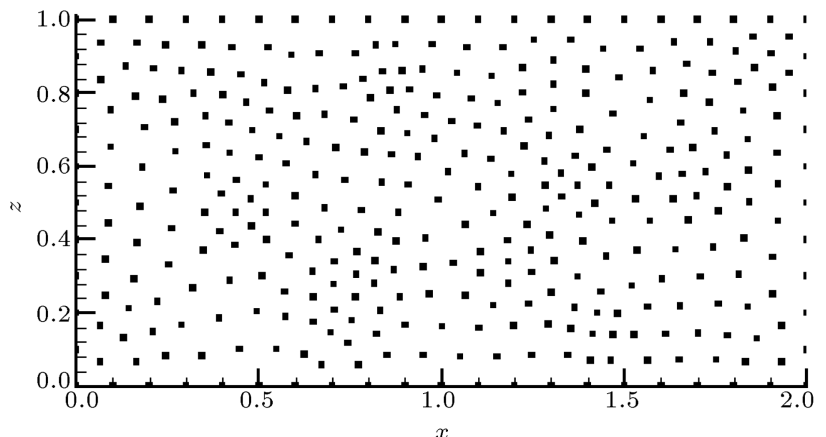

Figure 4. Domain discretization for Problem 1.

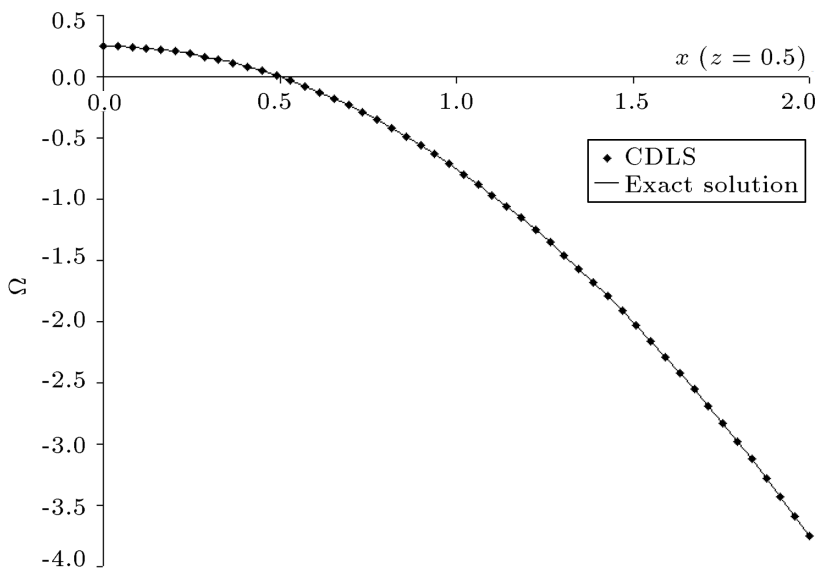

Figure 5. Comparison between numerical and analytical solutions for Problem 1.

case for the validation of the proposed numerical scheme. The exact solution to the governing equations can be obtained analytically by:

$$
\Omega(x, z)=-x^{2}+4 x z-z^{2}-2 x+z .
$$

In domain discretization, 355 nodes are used. The distribution of the nodes is shown in Figure 4. To compare analytical results with the numerical ones, the profile of $\Omega$ at section $z=0.5$ is depicted in Figure 5 for both solutions. Although a rather small number of nodes are used in the domain discretization, numerical results show good agreement with the exact solution.

\subsection{Problem 2}

The seepage problem beneath a small concrete dam is solved by considering two kinds of temperature boundary conditions. The geometry of the problem is shown in Figure 6. It is a typical problem in the soil mechanics discipline that should be considered carefully in the design procedure of the hydraulic structures. In this problem, an inclined impermeable bedrock is considered to show the simple domain discretization of a mesh-free method. In the first case (Case I), it is assumed that the soil layer is colder than the environment. In the second case (Case II), the temperature of the environment is considered colder

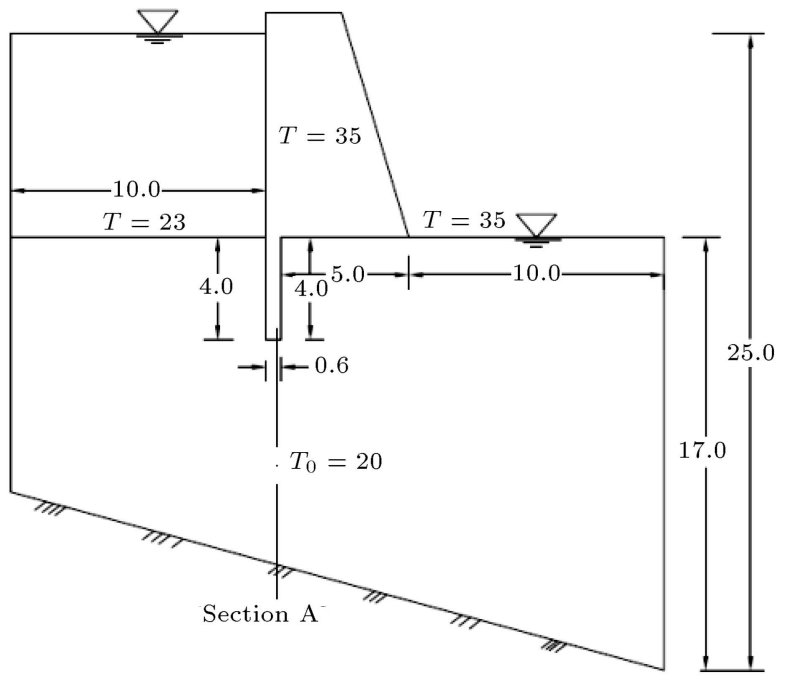

Figure 6. Geometry and boundary condition for Case I.

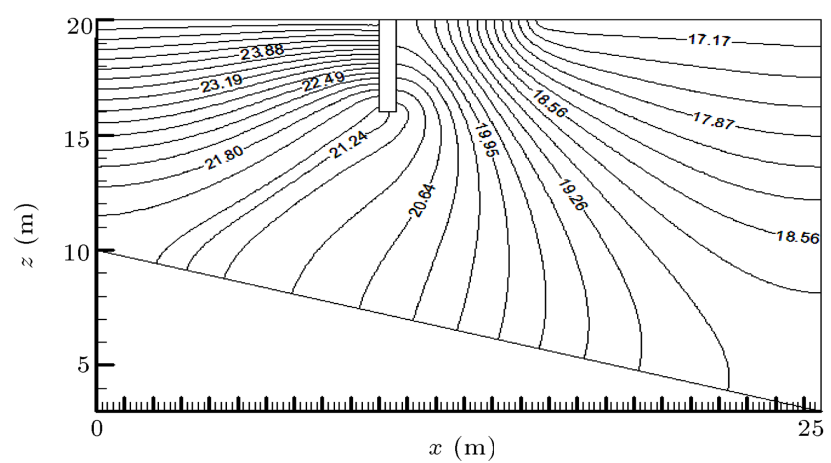

Figure 7. Equipotential lines without considering temperature effect.

than the initial temperature in the soil layer. For both cases, assume that the given temperatures at the boundaries do not change in the following 50 days. The permeability coefficient at $20^{\circ} \mathrm{C}$ and thermal diffusivity are chosen to be $0.879(\mathrm{~m} /$ day) and 0.08 $\left(\mathrm{m}^{2}\right.$.day $\left.{ }^{-1}\right)$ for the two cases, respectively. The initial and boundary conditions for the first case are shown in Figure 6. Equipotential lines without considering the temperature effects are shown in Figure 7 . As time passes, temperature distributes through the soil layer and changes the permeability values of any point in the soil. The temperature distributions for Case I, after 5, 10, and 50 days, are shown in Figures 8-10. In Figure 11, the variation of the permeability coefficient after 10 days is shown, which exhibits the relationship between the permeability coefficient and the temperature of each point. Since a mesh-free method is used in domain discretization, the permeability coefficient can be assigned at any point in the domain according to its calculated temperature. Figures $12-14$ show the resultant equipotential lines after passing 5, 10, and 50 days. To show the effect of the temperature on the value of the seepage, the horizontal velocity profile 


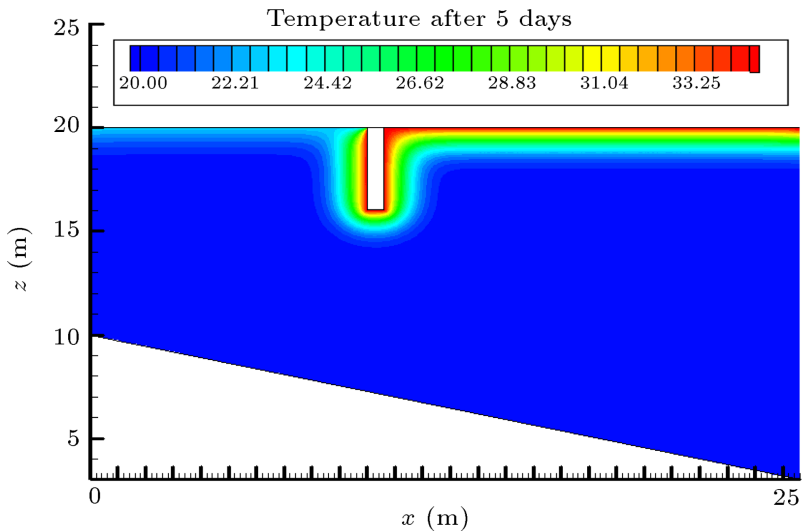

Figure 8. Temperature distribution after 5 days for Case I.

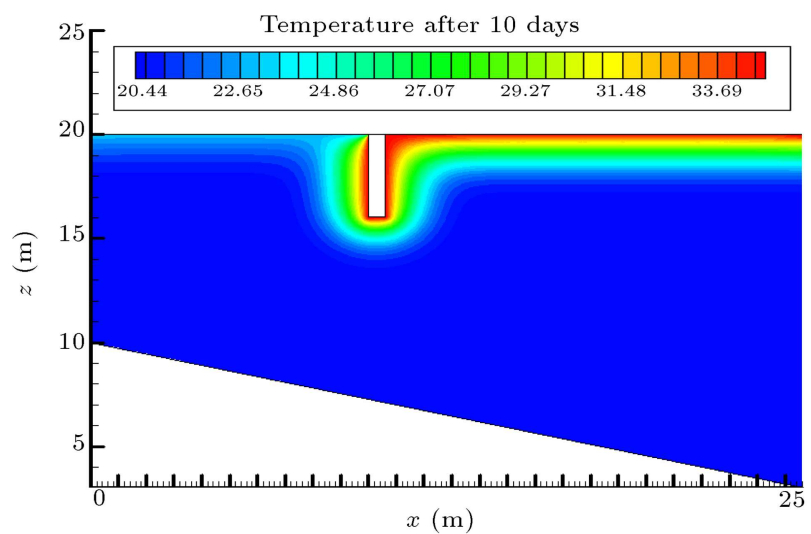

Figure 9. Temperature distribution after 10 days for Case I.

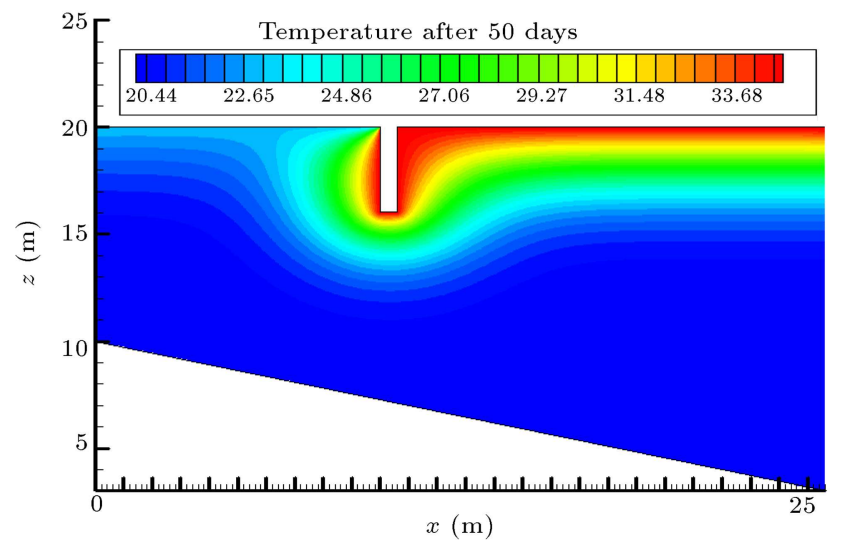

Figure 10. Temperature distribution after 50 days for Case I.

at Section $\mathrm{A}$ is calculated 5, 10, and 50 days after starting the simulation. For Case I, this comparison is shown in Figure 15. This Figure shows that as the temperature penetrates into the soil layer, the seepage value (discharge) reduces.

Now, assume that the soil initial temperature is higher than its boundaries. The new boundary conditions are shown in Figure 16. This phenomenon occurs usually in colder seasons. The temperature

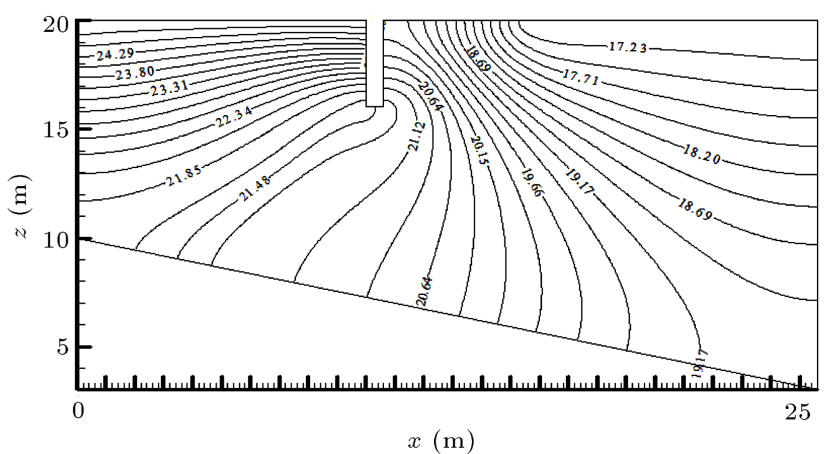

Figure 11. Equipotential lines after 5 days for Case I.

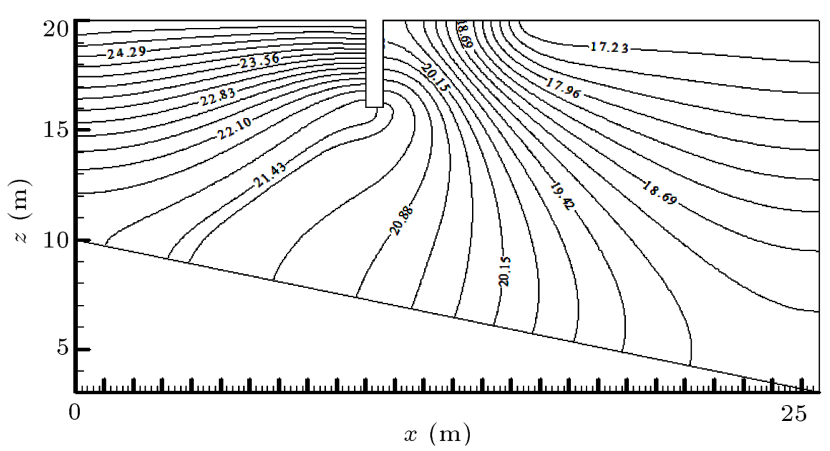

Figure 12. Equipotential lines after 10 days for Case I.

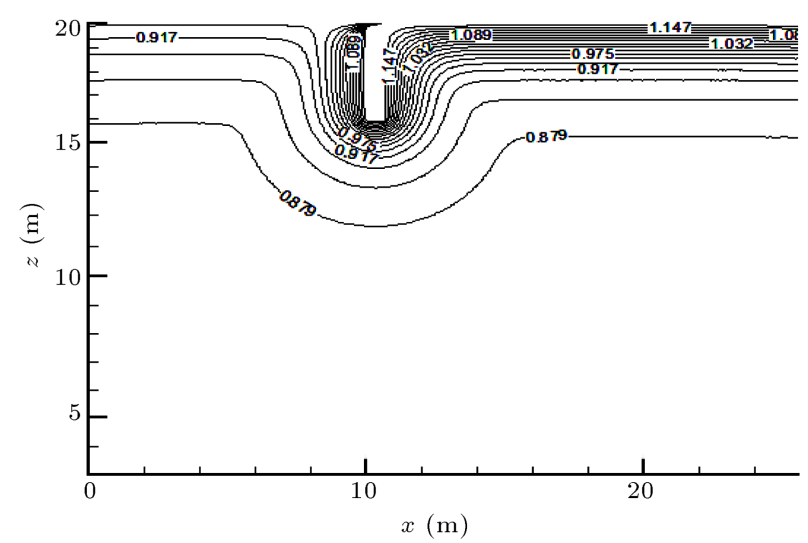

Figure 13. Distribution of the permeability coefficient after 10 days for Case I.

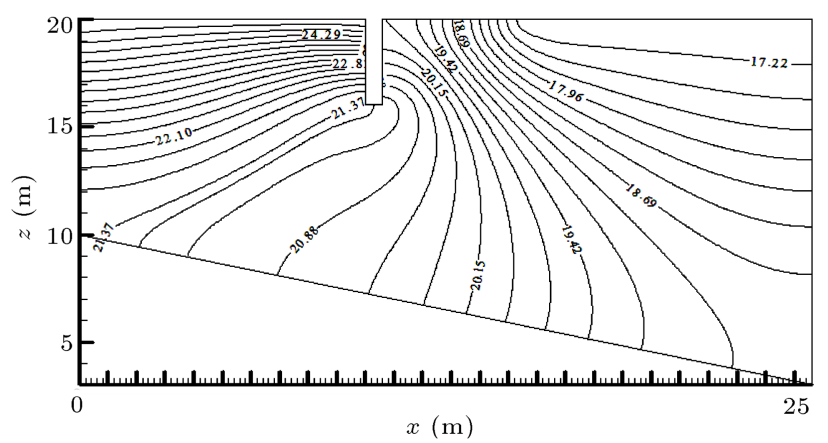

Figure 14. Equipotential lines after 50 days for Case I. 


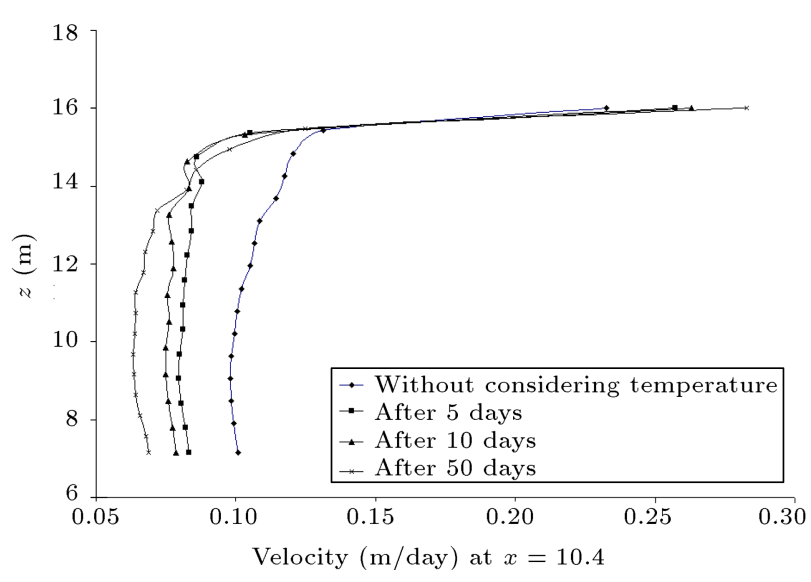

Figure 15. The horizontal velocity distribution at Section A, for Case I, after 5, 10 and 50 days.

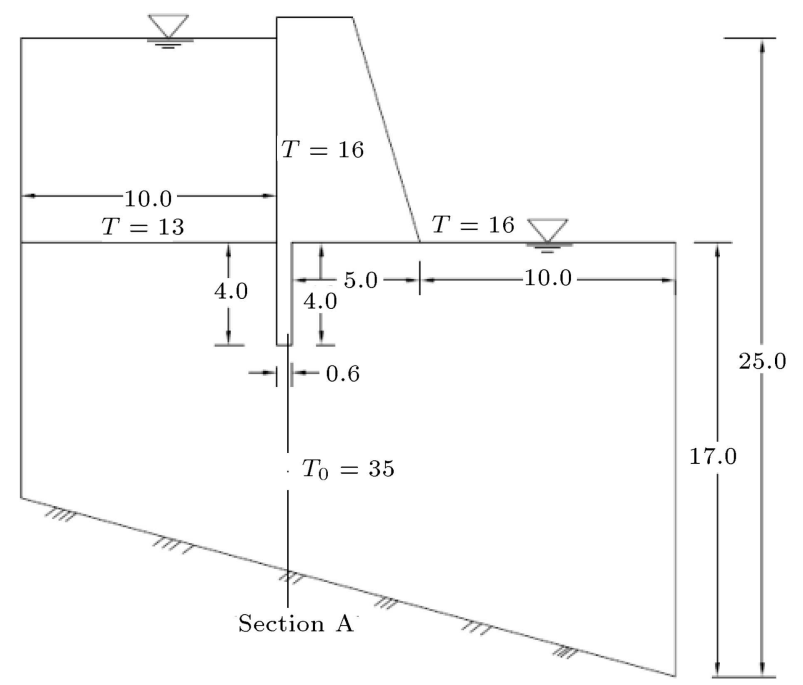

Figure 16. Geometry and boundary condition for Case II.

distributions after 5, 10, and 50 days are shown in Figures 17-19. As mentioned before, in the proposed methodology, the value of the permeability coefficient can be assigned to each discrete node and stored in a vector. The assigned values of this coefficient at a region near cut-off are shown in Figure 20. The equipotential lines for this case, 50 days after starting simulation, are shown in Figure 21. The horizontal velocity at Section A, for Case II, is shown in Figure 22. The significant differences between the top and lower parts of the velocity profile (especially after 50 days) are related to the gradient of the equipotential lines. A detailed investigation of Figure 21, reveals the higher gradient of the equipotential lines (which are closer) near $z=16 \mathrm{~m}$ (Figure 21 - circle (a)) than in the near area of the bed (Figure 21 - circle (b)). This difference derives from permeability gradient in $x$ direction resulted from the temperature distribution. Compared to Case I, in Case II, the seepage discharge increases as the soil loses its temperature.

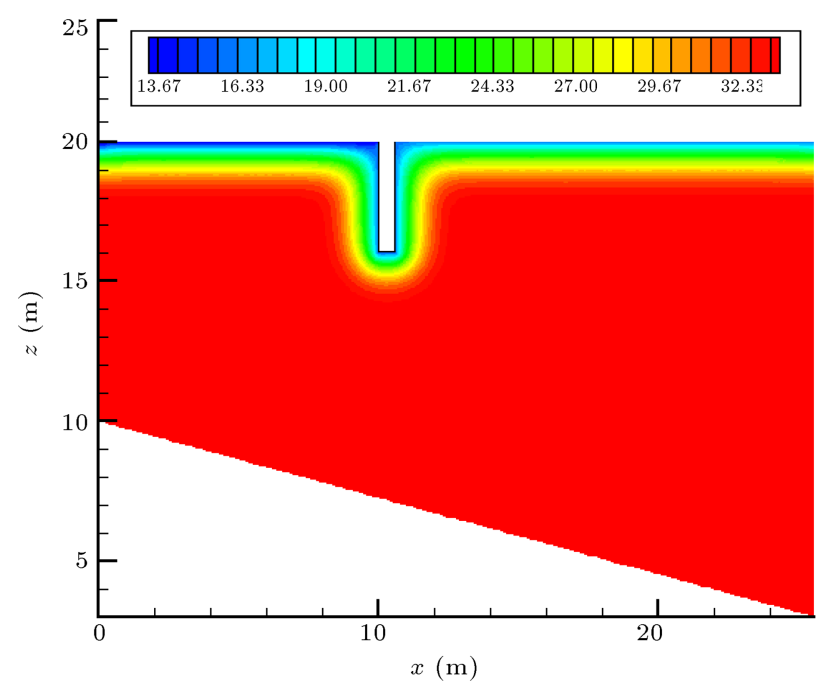

Figure 17. Temperature distribution after 5 days for Case II.

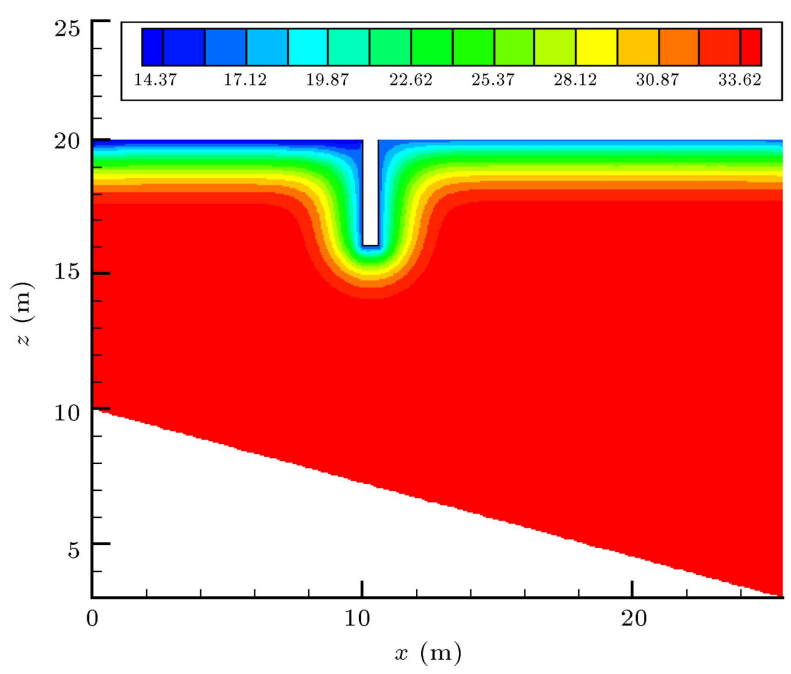

Figure 18. Temperature distribution after 10 days for Case II.

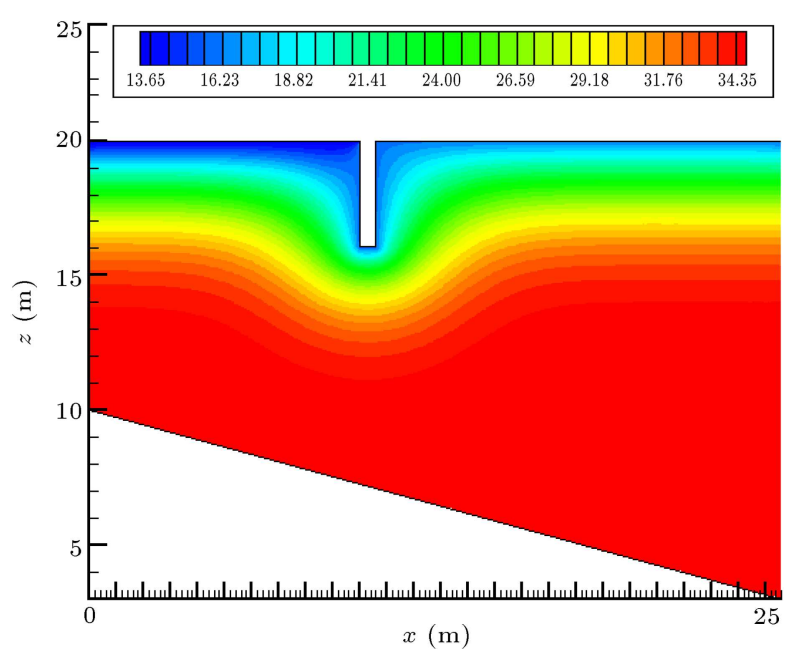

Figure 19. Temperature distribution after 50 days for Case II. 


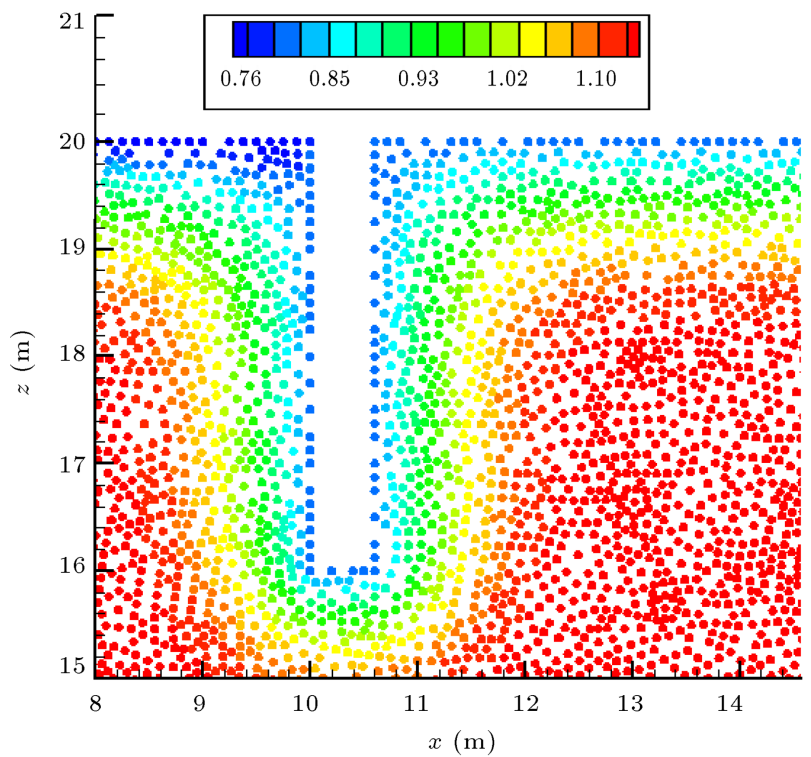

Figure 20. Assigned permeability coefficient for each node after 10 days for Case II.

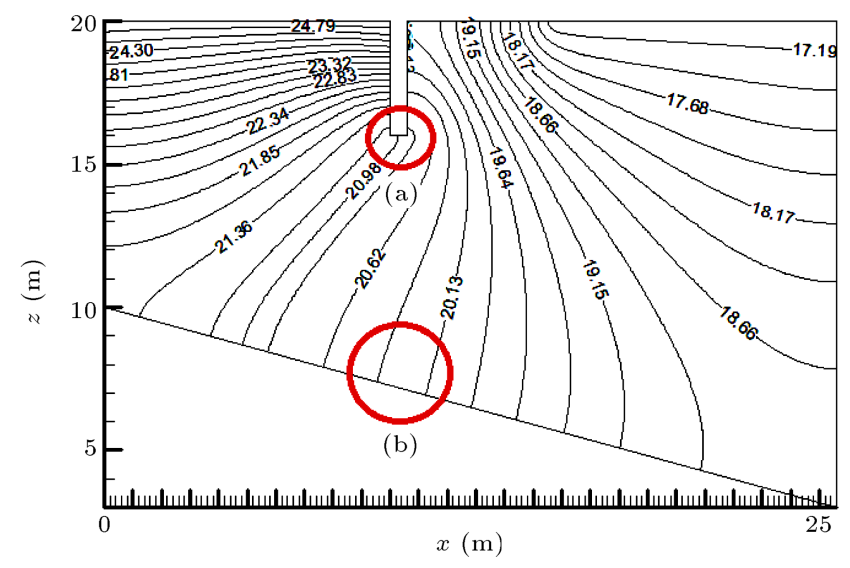

Figure 21. Equipotential lines after 50 days for Case II; higher gradient at $z=16 \mathrm{~m}$ (circle (a)) than lower gradient at $z=7 \mathrm{~m}$ (circle (b)).

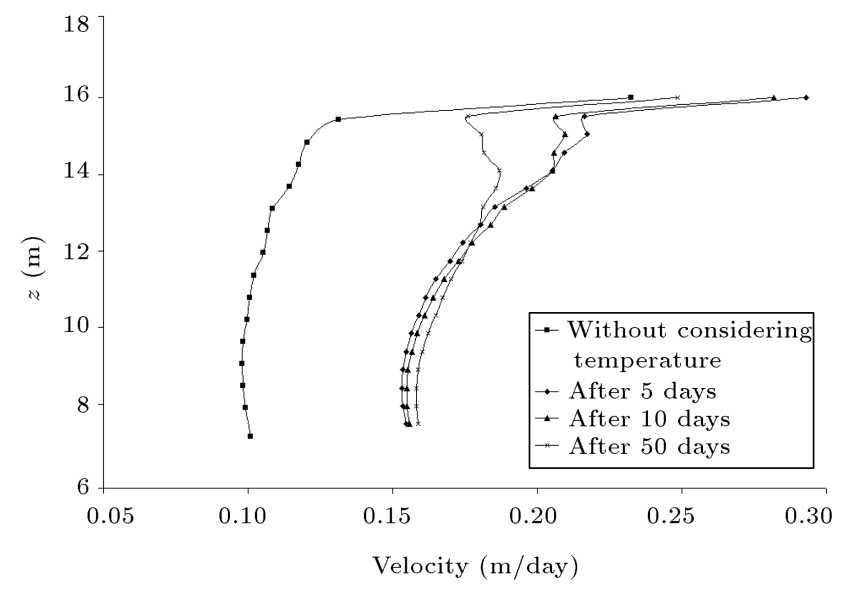

Figure 22. The horizontal velocity distribution at Section A, for Case II, after 5, 10 and 50 days.

\section{Conclusion}

Distribution of temperature in a soil layer makes it inhomogeneous since the coefficient of the permeability depends on the viscosity of water. This also depends on the temperature itself. By this interpretation, the permeability coefficients at any point in the soil vary according to temperature. In the collocated discrete least squares method, the coefficient of the permeability can be assigned to each node of the discretization point, while conventional numerical methods require interpolating this value on the surface of an element in the solution procedure. The variation of the permeability in the soil domain due to temperature distribution is calculated using the CDLS method. The permeability of zones with higher temperature possesses bigger permeability coefficient. In this paper, a simple matrix formulation is presented that makes the method easy for implementation and efficient in calculation. Results showed that the distribution of the permeability coefficient due to temperature has significant effects on the seepage problem. It is shown that the discharge value increases as a soil layer loses its temperature, and it decreases while the soil layer is getting warmer.

\section{References}

1. Farouki, T.O., Thermal Properties of Soils, United States Army Corps of Engineers (1981).

2. Putkonen, J. "Soil thermal properties and heat transfer processes near Ny-Alesund, northwestern Spitsbergen, Svalbard", Polar Research, 17(20), pp. 165-179 (1998).

3. Nicholson, P.G., Soil Improvement and Ground Modification Methods, Butterworth Heinemann, Elsevier Inc., USA (2015).

4. Zihms, S.G., Switzer, C., Karstunen, M., and Tarantino, A. "Understanding the effects of high temperature processes on the engineering properties of soils", Proceedings of the 18th International Conference on Soil Mechanics and Geotechnical Engineering, Paris, France, pp. 3427-3430 (2013).

5. Cho, W.J., Lee, J.O., and Chun, K.S. "The temperature effects on hydraulic conductivity of compacted bentonite", Applied Clay Science, 14, pp. 47-58 (1999).

6. Towhata, I., Kuntiwattanakul, P., Seko, I., and Ohishi, $\mathrm{K}$. "Volume change of clays induced by heating as observed in consolidation tests", Soils and Foundations, 33(4), pp. 170-183 (1993).

7. Villar, M.V. and Lioret, A. "Influence of temperature on the hydro-mechanical behaviour of a compacted bentonite", Applied Clay Science, 26(1/4), pp. 337-350 (2004).

8. Romero, E., Gens, A., and Lioret, A. "Temperature effects on the hydraulic behaviour of an unsaturated clay", Geotechnical and Geological Engineering, 19, pp. 311-332 (2001). 
9. Ye, W.M., Wan, M., Chen, B., Chen, Y.G., Cui, Y.J., and Wanf, J. "Temperature effects on the unsaturated permeability of the densely compacted GMZ01 bentonite under confined conditions", Journal of Engineering Geology, 126, pp. 1-7 (2012).

10. Stetyukha, V.A. "Numerical simulation of changes in the thermal condition of soils under the effect of channel change of a river bed", Power Technology and Engineering, 38(1), pp. 27-29 (2004).

11. Yu, W.B., Liu, W.B., Lai, Y.M., Chen, L., and Yi, $\mathrm{X}$. "Nonlinear analysis of coupled temperature seepage problem of warm oil pipe in permafrost regions of Northeast China", Applied Thermal Engineering, 70, pp. 988-995 (2014).

12. Yousefi, S., Noorzad, A., Ghaemian, M., and Kharaghani, S. "Seepage investigation of embankment dams using numerical modelling of temperature field", Indian Journal of Science and Technology, 6(8), pp. 5078-5082 (2013).

13. Cui, W., Gawecka, K.A., Potts, D.M., Taborda, D.M.G., and Zdravkovic, L. "Numerical analysis of coupled thermo hydraulic problems in geotechnical engineering", Geomechanics for Energy and the Environment, 6, pp. 22-34 (2016).

14. Fukuchi, T. "Numerical analyses of steady-state seepage problems using the interpolation finite difference method", Soils and Foundations, 56(4), pp. 608-626 (2016).

15. Belytschko, T. "Meshless methods: an overview and recent developments", Computer Methods in Applied Mechanics and Engineering, 139(1-4), pp. 3-47 (1996).

16. Liu, G.R., Mesh Free Methods: Moving Beyond the Finite Element Method, 1st, Ed. CRC Press, Boca Raton, USA (2002).

17. Liu, G.R. and Gu, Y.T., An Introduction to Meshless Methods and Their Programming, 1st, Ed. Springer Press, Berlin, Germany (2005).

18. Ding, H., Shu, C., Yeo, K.S., and Xu, D. "Development of least-square-based two-dimensional finite-difference schemes and their application to simulate natural convection in a cavity", Computer and Fluids, 33, pp. 137-157 (2004).

19. Afshar, M.H. and Lashckarbolok, M. "Collocated discrete least-squares (CDLS) meshless method: Error estimate and adaptive refinement", International Journal for Numerical Methods in Fluids, 56(10), pp. 19091928 (2008).

20. Afshar, M.H. and Shobeyri, G. "Efficient simulation of free surface flows with discrete least-squares meshless method using a priori error estimator", International Journal of Computational Fluid Dynamics, 24(9), pp. 349-367 (2010).

21. Firoozjaee, A.R. and Afshar, M.H. "Steady-state solution of incompressible Navier-Stokes equations using discrete least-squares meshless method", International Journal for Numerical Methods in Fluids, 67(3), pp. 369-382 (2010).

22. Lashkarbolok, M., Jabbari, E., and Westerweel, J. "A least squares based meshfree technique for the numerical solution of the flow of viscoelastic fluids: A node enrichment strategy", Engineering Analysis with Boundary Elements, 50, pp. 59-68 (2015).

23. Lashckarbolok, M. and Jabbari, E. "Collocated discrete least squares (CDLS) meshless method for the simulation of power-law fluid flows", Scientia Iranica, 20(2), pp. 322-328 (2013).

24. Lashckarbolok, M., Jabbari, E., and Vuik, K. "A node enrichment strategy in collocated discrete least squares meshless method for the solution of generalized Newtonian fluid flow", Scientia Iranica (A), 21(1), pp. 1-10 (2014).

25. Butcher, J.C., Numerical Methods for Ordinary Differential Equations, John Wiley, 2nd Edition (2008).

\section{Biographies}

Alireza Tabarsa received his $\mathrm{PhD}$ degree from Iran University of Science and Technology in the field of Soil Mechanics and Geotechnical Engineering. His research is focused on soil improvement. He has been an Assistant Professor in Golestan University since 2009.

Mohsen Lashkarbolok received his $\mathrm{PhD}$ from Iran University of Science and Technology in the field of Computational Fluid Dynamics. He was one of the key developers of the CDLS mesh-free method. He has been an Assistant Professor in Golestan University since 2013 . 